\title{
Genetic Diversity for Cold Tolerance at Seedling Stage in Rice (Oryza sativa L.) under Egyptian Conditions
}

\author{
W. H. Elgamal, M. A. A. El Sayed, E. A. Z. El Shamey* and G. B. Anis \\ Rice Research Department, Field Crops Research Institute, Agricultural Research \\ Center, Egypt.
}

\begin{abstract}
B REEDING rice for cold tolerance is one of the important ways for climate change facing, which attacks a large amount of the cultivated areas all over the world countries. This study was perfomed at Sakha Agricultural Research Station during two rice growing seasons 2015 and 2016 to evaluate some rice accessions which belong to different rice types for the ability of cold tolerance in seedling stage at early growing season under Egyptian conditions. The 40 rice accessions used in this study were evaluated at two early dates of sowing $1^{\text {st }}$ March and $1^{\text {st }}$ April as a cold condition, in addition to control sowing date at $1^{\text {st }}$ May. Twenty six SSR markers related to cold tolerance at seedling stage were used in order to determine the genetic diversity and polymorphism among the studied genotypes. Low temperature degree condition significantly decreased the mean values of the all studied traits for all accessions compared with the control condition. Significant differences were observed among the studied genotypes under both different growing conditions. Japonica varieties are found to be more tolerant than Indica varieties. SSR markers RM265, RM324, RM163 and RM270 showed highly polymorphism among the studied rice accessions and more over differentiate them to different groups based on their ability for cold tolerance. Giza 177, Sakha 102 and Sakha 104 out of the Egyptian rice genotypes showed a good performance at low temperature degrees condition in early growing season. The genotypes CT6749-36-7-2-M-M, Milyang 80, PSRM1-17-4B-13, Ryong Sung 25 can be used as a donor for cold tolerance in rice breeding program.
\end{abstract}

Keywords: Rice, Evaluation, Cold tolerance, Seedling stage, SSR markers

\section{Introduction}

Rice (Oryza sativa L.) is one of the major staple food crops and critical to food security for billions of people around the world. Egypt is the largest rice producer in the Near East region. The rice cultivated area in Egypt is about 0.6 million ha annually, all is under irrigation eco- system (Kim et al., 2011). Rice cultivation takes place at Egyptian Nile delta especially in the northern part. Constantly changing climatic conditions in Egypt and around the world demand constant efforts to understand and adapt to environmental challenges and developing new varieties for sustainable crop production. The challenge is even greater for crops such as rice (Oryza sativa $\mathrm{L}$.), which is the staple of more than half the world's population and grown under diverse environmental conditions. So, rice breeders considered to be the most pragmatic approach for better yield against environmental challenge and population increase. One important strategy for increasing crop productivity is to minimize losses due to biotic and abiotic stresses by developing more stress-tolerant varieties (Boyer, 1982 and Khush, 1999).

Intraspecific genetic variability for cold tolerance has been reported in rice and according to reports Japonica cultivars are more tolerant to low temperatures than Indica cultivars (Andaya and Mackill, 2003). Genetic variability for cold tolerance has been evaluated by researchers at germination, seedling and reproductive stages in rice breeding programs. Cold stress in rice leads to poor germination and stunted growth of seedlings, delays flowering, lengthens the growth cycle, reduces tillering, increases plant mortality (Mackill and lei, 1997) and causes significant yield loss in rice production (Murai et al., 1991 and Nagasawa et al., 1994), thus enhancing cold tolerance through the use of a diverse germplasm 
of Japonica rice is a very important objective in rice breeding. To enhance cold tolerance in rice, it is therefore imperative to exchange and evaluate various rice germplasm from temperate rice-growing countries for cold stress at various growth stages in key Japonica rice cultivation sites. Elite cold-tolerant rice germplasm also need to be crossed with a diverse set of elite cultivars from each country. However, there are limited genetic resources for the improvement of cold tolerance in temperate Japonica rice cultivars. Some tropical Japonica cultivars have been reported to exhibit cold tolerance (Glaszmann et al., 1990 and Saito et al., 2001).

Various methods can be used to create cold injury environments to evaluate cold tolerance at the booting and reproductive stages in rice. However, screening several materials for cold tolerance is difficult, more so in producing reliable results. Therefore, it is necessary to use a reproducible phenotyping method to measure tolerant and sensitive traits under cold stress and apply improved QTL analysis methods to identify effective QTLs for cold tolerance at the reproductive stage. SSR markers produce a high frequency of polymorphic alleles in rice and are suitable for evaluating genetic diversity among closely related rice cultivars, including Japonica rice (Suh et al., 2004). Several studies aim to identify QTLs conferring cold tolerance at early growth stage by using SSR molecular markers including cold tolerance at the seedling, tillering stage and the growth ability of seedling under low temperatures in rice. The detected QTLs associated with the cold tolerance in early growth period will provide the effective genetics evidences for the molecular marker assisted breeding (Qia et al., 2004, Xu et al., 2008 and Ye et al., 2010).

Problems for farmers leading to loss of production as well as reduced milling quality caused by water deficit at late June and high temperature at August. Thus, development of rice varieties tolerant to low temperatures at the early stages will allow farmers to plant their own season crop in April beginning at least. Hence, they able to avoid the water deficit at tillering, and panicle initiation stages and high temperature at ripening stage exhibited high negative effect on grain quality. This study aims to evaluate some introduced genotypes and Egyptian commercial varieties in addition to promising lines using phenotyping and genotyping characterization to identify the best genetic sources for cold tolerance breeding program.

J. Sus. Agric .Sci. 44, No.2 (2018)

\section{Materials and Methods}

Plant materials and phenotyping

A total of 40 rice accessions belong to the three rice subspecies; Japonica, Indica and Indica/Japonica used at this study to evaluate its ability for cold tolerance at seedling stage under early growing season. The experiment was conducted at the green house of Rice Research \& Training Center (RRTC), Sakha, Egypt. The rice germplasm accessions were originally procured from the International Rice Research Institute (IRRI), Philippines and Rice Research \& Training Center (RRTC), Egypt (Table 1). The grains of the 40 rice accessions were sown in plastic seedling plates with a size of $60 \mathrm{~cm} \times 30 \mathrm{~cm} \times 5 \mathrm{~cm}$, supplied with nutrient soil and managed by the recommended agricultural practices, at two early dates of sowing $1^{\text {st }}$ March and $1^{\text {st }}$ April as a cold stress, in addition to control sowing date at $1^{\text {st }}$ May. Randomized complete block design (RCBD) with three replicates was the experimental design and repeated in two rice growing seasons of 2015 and 2016. During the two sowing dates the degrees of temperature were lower than normal condition for rice germination and growing, the temperature degrees in Table 2 were recorded by Rice Research and Training Center meteorological station, Sakha, Kafr Elsheikh, Egypt. When the seedlings were at 30 days age, five seedling observations were recorded: germination $\%$, germination index, seedling height, root length and number of leaves/seedling. The cold reduction index used to determine the accessions ability to tolerate the cold effects and calculate as follow: [(the value under normal conditions - the value under stress)/ the value under normal conditions] $]^{*} 100$.

\section{Statistical analysis}

The obtained data were statistically analyzed for analysis of variance according to Gomez and Gomez (1984) by using computer statistical software of MSTAT-C. Treatment means were compared by LSD. Cluster analysis was analyzed by Paleontological statistics PAST software, version 2.17 (Saitou and Nei, 1987).

\section{SSR markers analysis}

The fresh leaves of 40 rice accessions were sampled for DNA extraction according to modified CTAB (Cetyl Try Methyl Ammonium Bromide) method (Rogers and Bendich, 1988). A total of twenty six SSR markers associated with cold tolerance from previous studies were used in the polymorphism survey. PCR was performed as follow: The $10-\mu \mathrm{LPCR}$ reaction mixture contained 
$1 \mathrm{x}$ buffer, $0.2 \mu \mathrm{M}$ of each primer, $50 \mu \mathrm{M}$ of dntps, 0.5 unit of Taq polymerase (Tiangen Company, Beijing, china), and $10 \mathrm{ng}$ of genomic DNA as a template. The thermal cycler was programmed for a first denaturation step of $4 \mathrm{~min}$ at $94^{\circ} \mathrm{C}$, followed by 30 cycles, each of $94^{\circ} \mathrm{C}$ for $30 \mathrm{~s}, 55^{\circ}$ for $30 \mathrm{~s}$ and $72^{\circ} \mathrm{C}$ for $30 \mathrm{~s}$ and final extension step of 5 min at $72^{\circ} \mathrm{C}$. The PCR products were separated on $8.0 \%$ non-denaturing polyacrylamide gel and detected using the silver staining method. Generated DNA bands were analyzed and scored 1 for the presence- or 0 for the absence of allele. Four SSR markers (RM 265, RM324, RM163 and RM270) were highly polymorphic among the studied genotypes which used for genotyping are listed in table 3 (Jiang et al., 2011 and Yang et al., 2013) and these markers located on chromosomes $1,2,5$ and 12 , respectively.

\section{Resultes and Discussion}

Analysis of variance

Analysis of variance showed that the mean square values due to years $(\mathrm{Y})$ were significant and highly significant for all studied traits except germination index. Genotypes $(\mathrm{G})$ and treatments (T) were highly significant for all studied traits, while all possible interactions ( $\mathrm{Y} \times \mathrm{G}, \mathrm{Y} \times \mathrm{T}, \mathrm{G} \times$ $\mathrm{T}$ and $\mathrm{Y} x \mathrm{G} \times \mathrm{T}$ ) showed highly significant for all studied traits except number of leaves /seedling (Table 4). These results indicating for high variances between genotypes in addition to effective treatments by sowing date as a different temperature under Egyptian conditions. On other hand, a little effect of years under Egyptian conditions. Abdelmaksoud et al., 2007; Elgamal, 2013 and Elgamal et al., 2015 reported that the effect of years is not highly significant on rice growing under Egypt conditions.

TABLE 1. Origin and type of the 40 rice accessions used in this study

\begin{tabular}{|c|c|c|c|c|c|c|c|}
\hline No. & Accession name & Origin & type & No. & Accession name & Origin & Type \\
\hline 1 & CT6749-36-7-2-M-M & Chile & Japonica & 21 & Giza 181 & Egypt & Indica \\
\hline 2 & H270-30-2-1 & Argentina & Indica & 22 & Giza 182 & Egypt & Indica \\
\hline 3 & Barakat (K787-13) & India & Indica & 23 & Sakha 101 & Egypt & Japonica \\
\hline 4 & Hexi 25 & China & Japonica & 24 & Sakha 102 & Egypt & Japonica \\
\hline 5 & HSC16 & Hungary & Indica & 25 & Sakha 103 & Egypt & Japonica \\
\hline 6 & IR63347-AC201-1 & Philippines & Indica & 26 & Sakha 104 & Egypt & Japonica \\
\hline 7 & L10573(ACC68106) & USA & Japonica & 27 & Sakha 105 & Egypt & Japonica \\
\hline 8 & LOTO & Italy & Indica & 28 & Sakha 106 & Egypt & Japonica \\
\hline 9 & Milyang 80 & Korea & Indica /Japonica & 29 & E. Yasmine & Egypt & Indica \\
\hline 10 & PEGASO & Italy & Indica & 30 & GZ4328-1-2-1-3 & Egypt & Indica/ Japonica \\
\hline 11 & PSRM1-17-4B-13 & Philippines & Indica & 31 & GZ4362-34-2-1-3 & Egypt & Indica/ Japonica \\
\hline 12 & Ryong Sung 25 & Korea & Japonica & 32 & GZ4461-4-2-3-1 & Egypt & Indica/ Japonica \\
\hline 13 & SOHAECHAL & Korea & Japonica & 33 & GZ4514-3-1-3-1 & Egypt & Indica/ Japonica \\
\hline 14 & ZHONG JIA & China & Indica /Japonica & 34 & GZ9057-34-6-1-3-2(Giza179) & Egypt & Indica/ Japonica \\
\hline 15 & ZHONG JU3 & China & Japonica & 35 & GZ9057-34-6-1-3-3 & Egypt & Indica/ Japonica \\
\hline 16 & Giza 171 & Egypt & Japonica & 36 & GZ9523-2-1-1-1 & Egypt & Indica/ Japonica \\
\hline 17 & Riho & Egypt & Japonica & 37 & GZ9577-4-1-1 & Egypt & Indica/ Japonica \\
\hline 18 & Giza 175 & Egypt & Japonica & 38 & СТ9506-44-2-1-1-M-3-4p-M-1 & Colombia & Indica \\
\hline 19 & Giza 177 & Egypt & Japonica & 39 & СТ9882-16-4-2-3-2-p-M & Colombia & Indica \\
\hline 20 & Giza 178 & Egypt & Indica/ Japonica & 40 & СТ9505-17-3-1-1-M-1-3p-M & Colombia & Indica \\
\hline
\end{tabular}

TABLE 2. Average of temperature degrees between day and night for growing dates

\begin{tabular}{ccccccc}
\hline \multirow{2}{*}{ Month } & \multicolumn{2}{c}{$\mathbf{2 0 1 5}$} & \multicolumn{2}{c}{$\mathbf{2 0 1 6}$} \\
\cline { 2 - 6 } & $\mathbf{1}^{\text {st }}-\mathbf{1 0}^{\text {th }}$ & $\mathbf{1 1}^{\text {th }}-\mathbf{2 0}^{\text {th }}$ & $\mathbf{2 1}^{\text {th }}-\mathbf{3 0}^{\text {th }}$ & $\mathbf{1}^{\text {st }}-\mathbf{1 0}^{\text {th }}$ & $\mathbf{1 1}^{\text {th }}-\mathbf{2 0}^{\text {th }}$ & $\mathbf{2 1}^{\text {th }}-\mathbf{3 0}^{\text {th }}$ \\
\hline March & $17.35^{\circ} \mathrm{C}$ & $16.23^{\circ} \mathrm{C}$ & $18.38^{\circ} \mathrm{C}$ & $17.55^{\circ} \mathrm{C}$ & $16.31{ }^{\circ} \mathrm{C}$ & $18.39{ }^{\circ} \mathrm{C}$ \\
April & $19.80^{\circ} \mathrm{C}$ & $20.98^{\circ} \mathrm{C}$ & $23.77^{\circ} \mathrm{C}$ & $19.30{ }^{\circ} \mathrm{C}$ & $18.60{ }^{\circ} \mathrm{C}$ & $21.46{ }^{\circ} \mathrm{C}$ \\
May & $25.67^{\circ} \mathrm{C}$ & $23.67^{\circ} \mathrm{C}$ & $25.73{ }^{\circ} \mathrm{C}$ & $23.21{ }^{\circ} \mathrm{C}$ & $24.35^{\circ} \mathrm{C}$ & $25.91{ }^{\circ} \mathrm{C}$ \\
\hline
\end{tabular}


TABLE 3. List and sequences of polymorphic primers

\begin{tabular}{cccc}
\hline No & Name & Forward sequence & Reverse sequence \\
\hline 1 & RM265 & 5'-CGAGTTCGTCCAAGTGAGC-3' & 5'-CATCCACCATTCCACCAATC-3' \\
2 & RM324 & 5'-CTGATTCCACACACTTGTGC-3' & 5'-GATTCCACGTCAGGATCTTC-3' \\
3 & RM163 & 5'-ATCCATGTGCGCCTTTATGAGGA-3' & 5'-CGCTACCTCCTTCACTTACTAGT-3' \\
4 & RM270 & 5'-GGCCGTTGGTTCTAAAATC-3' & 5'-TGCGCAGTATCATCGGCGAG-3' \\
\hline
\end{tabular}

TABLE 4 . Analysis of variance and the mean squares of years, treatments and genotypes, and the inter actions for all studied traits

\begin{tabular}{|c|c|c|c|c|c|c|}
\hline S. O. V. & D.F. & G\% & G. I & S.H & R. L & N.L/S \\
\hline Reps (R) & 2 & 3.890 & 0.029 & 0.094 & 1.106 & 4.213 \\
\hline Years (Y) & 1 & $764.0 * *$ & 0.001 & $0.350^{* *}$ & $3.199 * *$ & $3.627 *$ \\
\hline Treatments $(\mathrm{T})$ & 2 & $7637 * *$ & $9.474 * *$ & $1212 * *$ & $623.3 * *$ & $188.6^{* *}$ \\
\hline Genotypes (G) & 39 & $2088 * *$ & $0.121 * *$ & $8.525 * *$ & $6.205 * *$ & $5.680^{* *}$ \\
\hline Y.T & 2 & $734.2 * *$ & $0.002 *$ & $0.022 * *$ & $0.575^{* *}$ & 0.520 \\
\hline Y.G & 39 & $89.97 * *$ & $0.004 * *$ & $0.265 * *$ & $0.162 * *$ & 1.088 \\
\hline T.G & 78 & $842.1 * *$ & $0.038 * *$ & $2.636^{* *}$ & $2.504 * *$ & 1.004 \\
\hline Y.T.G & 78 & $95.18 * *$ & $0.003 * *$ & $0.262 * *$ & $0.168 * *$ & 0.770 \\
\hline Residual & 478 & 7.663 & 0.001 & 0.020 & 0.045 & 0.784 \\
\hline
\end{tabular}

Note: *,** significant at 0.05 and 0.01 levels of probability, respectively.

G \%: germination percentage, G.I: germination index, S.H: seedling height, R.L: root length and N.L/S: number of leaves / seedling. Treatments are different temperature degrees as a three different dates of sowing.

\section{Phenotyping characterization}

Low temperature effects on rice seedling growth such as; poor and delayed germination, retarded seedling growth, leaves discoloration and inhibited rooting (Lee 2001; Shu and Jayab 2001). Results presented in Tables 5 - 7 showed that there is no particular accession recorded the highest values for all studied traits at the different levels of low temperature along with the control during the two growing seasons. On the other hand, some of the studied genotypes, i.e. CT6749-36-7-2-M-M (1), Milyang 80 (9), Ryong Sung 25 (12), CT9505-17-3-1-1-M-13p-M (40), Giza 177 (19), Sakha 102 (24), Sakha 104 (26) showed good results for most studied traits.

For Germination \% the accession Giza 177(19) followed by Sakha 104 (26), Giza 171(16), Sakha 101(23), Sakha 102 (24) and Riho (17) scored the highest values under the lowest cold degree for the two growing seasons with range from $83.67 \%$ to 69.00 $\%$. Regarding to germination index the Accessions Milyang 80 (9), Giza 177 (19), Sakha 104 (26), Sakha 102 (24), Ryong Sung 25 (12) and Sakha 103(25) showed the best performance after germination under the lowest degree of temperature for the two growing seasons and the values ranged from 0.80 to 0.70 .

With respect to, seedling height the accessions CT9505-17-3-1-1-M-1-3p-M (40), Zhong Jia(14),
CT6749-36-7-2-M-M (1), Giza 177(19), Giza 171(16) and Sakha 102 (24) had the highest seedling under the hardest stress for the two grown seasons with values $5,4.5,4,3.83,3.83$ and $3.67 \mathrm{~cm}$ respectively. While the accessions CT9505-173-1-1-M-1-3p-M (40), CT9882-16-4-2-3-2-p-M (39), CT6749-36-7-2-M-M (1), Giza 177 (19), PSRM1-17-4B-13(11) and Riho(17) showed the deepest root length under the coldest growing date for the two growing seasons with values ranged from 5 to $3.17 \mathrm{~cm}$. In relation to number of leaves per seedling the accessions Milyang 80 (9), Sakha 104 (26), Ryong Sung 25 (12), CT6749-36-72-M-M (1), Giza 177 (19) and Sakha 102 (24) recorded the highest number of leaves under cold stress with values ranged from 3.66 to 2.66. These results indicated that these accessions have a good ability for growing at early growing dates when the temperature degrees are under the normal levels, and it could be conducted that these accessions have a cold tolerance trait. In order to emphases the cold tolerant accessions by using cold reduction index, the accessions CT6749-36-7-2-M-M, H270-30-21, Milyang 80, PSRM1-17-4B-13, Ryong Sung 25, Giza 177, Sakha102, Sakha 104, CT9882-16-4-23-2-p-M (Table 8) gave the lowest reduction index that means these accessions haven't been affected by cold stress. 
TABLE 5. The mean performance of accessions for three sowing dates at two years of 2015 and 2016 for germination percentage and germination index

\begin{tabular}{|c|c|c|c|c|c|c|c|c|c|c|c|c|}
\hline \multirow{3}{*}{ Genotypes } & \multicolumn{6}{|c|}{ Germination (\%) } & \multicolumn{6}{|c|}{ Germination Index } \\
\hline & \multicolumn{3}{|c|}{2015} & \multicolumn{3}{|c|}{2016} & \multicolumn{3}{|c|}{2015} & \multicolumn{3}{|c|}{2016} \\
\hline & $1^{\text {st }}$ March & $\begin{array}{c}1^{\text {st }} \\
\text { April }\end{array}$ & $\begin{array}{c}1^{\text {st }} \\
\text { May }\end{array}$ & $\begin{array}{c}1^{\text {st }} \\
\text { March }\end{array}$ & $\begin{array}{c}1^{\text {st }} \\
\text { April }\end{array}$ & $\begin{array}{c}\mathbf{1}^{\mathrm{st}} \\
\text { May }\end{array}$ & $1^{\text {st }}$ March & $\begin{array}{c}1^{\text {st }} \\
\text { April }\end{array}$ & $\begin{array}{c}1^{\text {st }} \\
\text { May }\end{array}$ & $1^{\text {st }}$ March & $\begin{array}{c}1^{\text {st }} \\
\text { April }\end{array}$ & $\begin{array}{c}1^{\text {st }} \\
\text { May }\end{array}$ \\
\hline 1 & 36.00 & 73.00 & 91.67 & 41.33 & 72.00 & 92.00 & 0.66 & 0.80 & 0.96 & 0.66 & 0.69 & 0.98 \\
\hline 2 & 11.03 & 78.00 & 91.33 & 20.30 & 75.00 & 91.33 & 0.60 & 0.82 & 0.94 & 0.60 & 0.80 & 0.94 \\
\hline 3 & 37.33 & 73.00 & 83.00 & 38.33 & 71.00 & 85.33 & 0.20 & 0.40 & 0.90 & 0.40 & 0.60 & 0.90 \\
\hline 4 & 24.33 & 83.33 & 90.67 & 31.00 & 73.00 & 92.00 & 0.30 & 0.40 & 0.94 & 0.30 & 0.60 & 0.94 \\
\hline 5 & 24.67 & 75.33 & 81.33 & 29.33 & 72.00 & 81.33 & 0.20 & 0.50 & 0.84 & 0.33 & 0.50 & 0.84 \\
\hline 6 & 15.00 & 91.33 & 93.33 & 43.33 & 88.30 & 93.33 & 0.40 & 0.60 & 0.90 & 0.40 & 0.60 & 0.90 \\
\hline 7 & 70.00 & 81.33 & 91.00 & 66.66 & 80.00 & 91.00 & 0.30 & 0.40 & 0.92 & 0.33 & 0.40 & 0.92 \\
\hline 8 & 17.30 & 60.33 & 90.33 & 31.33 & 60.00 & 90.33 & 0.30 & 0.30 & 0.88 & 0.30 & 0.40 & 0.88 \\
\hline 9 & 42.33 & 63.67 & 81.33 & 42.33 & 61.00 & 83.33 & 0.80 & 0.80 & 0.93 & 0.80 & 0.80 & 0.96 \\
\hline 10 & 20.30 & 57.67 & 63.67 & 35.33 & 60.00 & 65.66 & 0.40 & 0.60 & 0.68 & 0.40 & 0.60 & 0.76 \\
\hline 11 & 33.67 & 86.67 & 88.33 & 36.67 & 85.30 & 88.33 & 0.60 & 0.60 & 0.90 & 0.60 & 0.66 & 0.90 \\
\hline 12 & 48.00 & 75.67 & 93.33 & 49.33 & 75.00 & 96.67 & 0.71 & 0.88 & 0.94 & 0.70 & 0.90 & 0.95 \\
\hline 13 & 37.67 & 77.33 & 78.33 & 41.67 & 75.33 & 79.33 & 0.46 & 0.70 & 0.84 & 0.46 & 0.70 & 0.84 \\
\hline 14 & 22.00 & 80.00 & 82.33 & 43.66 & 78.00 & 82.33 & 0.36 & 0.80 & 0.88 & 0.36 & 0.80 & 0.88 \\
\hline 15 & 49.27 & 75.33 & 74.00 & 50.67 & 75.33 & 77.33 & 0.44 & 0.80 & 0.84 & 0.44 & 0.80 & 0.84 \\
\hline 16 & 79.67 & 81.33 & 86.33 & 78.33 & 82.00 & 86.33 & 0.50 & 0.84 & 0.90 & 0.50 & 0.84 & 0.90 \\
\hline 17 & 72.00 & 95.33 & 97.67 & 69.00 & 92.30 & 97.67 & 0.60 & 0.80 & 0.90 & 0.60 & 0.80 & 0.90 \\
\hline 18 & 33.00 & 93.33 & 100.00 & 55.33 & 85.70 & 97.66 & 0.40 & 0.68 & 0.88 & 0.40 & 0.68 & 0.88 \\
\hline 19 & 83.67 & 91.33 & 94.33 & 80.33 & 90.00 & 94.33 & 0.74 & 0.90 & 0.97 & 0.75 & 0.80 & 0.97 \\
\hline 20 & 39.67 & 86.00 & 74.00 & 40.00 & 85.00 & 74.00 & 0.65 & 0.80 & 0.93 & 0.65 & 0.80 & 0.93 \\
\hline 21 & 13.33 & 78.00 & 85.33 & 25.00 & 79.00 & 85.33 & 0.18 & 0.60 & 0.98 & 0.41 & 0.66 & 0.98 \\
\hline 22 & 35.00 & 80.00 & 75.33 & 54.66 & 77.66 & 75.33 & 0.21 & 0.70 & 0.96 & 0.33 & 0.70 & 0.96 \\
\hline 23 & 78.00 & 65.00 & 82.67 & 78.33 & 66.00 & 82.67 & 0.18 & 0.66 & 0.97 & 0.33 & 0.66 & 0.97 \\
\hline 24 & 74.33 & 90.67 & 95.00 & 73.33 & 90.00 & 95.00 & 0.72 & 0.88 & 0.96 & 0.72 & 0.88 & 0.96 \\
\hline 25 & 67.33 & 90.67 & 100.00 & 70.00 & 85.00 & 98.66 & 0.70 & 0.80 & 0.98 & 0.70 & 0.76 & 0.98 \\
\hline 26 & 81.67 & 83.33 & 91.67 & 80.00 & 84.00 & 91.67 & 0.72 & 0.78 & 0.98 & 0.74 & 0.81 & 0.98 \\
\hline 27 & 64.33 & 84.67 & 96.67 & 61.00 & 82.00 & 96.67 & 0.73 & 0.60 & 0.94 & 0.60 & 0.66 & 0.94 \\
\hline 28 & 49.67 & 61.67 & 62.00 & 48.66 & 60.00 & 62.00 & 0.70 & 0.80 & 0.97 & 0.66 & 0.80 & 0.97 \\
\hline 29 & 15.30 & 60.33 & 58.00 & 32.33 & 62.00 & 66.66 & 0.12 & 0.60 & 0.90 & 0.33 & 0.60 & 0.90 \\
\hline 30 & 59.30 & 57.67 & 67.33 & 52.31 & 55.70 & 67.33 & 0.44 & 0.70 & 0.94 & 0.44 & 0.70 & 0.94 \\
\hline 31 & 59.33 & 46.00 & 70.67 & 51.33 & 48.30 & 70.67 & 0.44 & 0.60 & 0.92 & 0.44 & 0.60 & 0.92 \\
\hline 32 & 24.33 & 52.67 & 56.33 & 38.70 & 55.00 & 62.33 & 0.50 & 0.80 & 0.94 & 0.50 & 0.80 & 0.94 \\
\hline 33 & 59.33 & 68.67 & 69.33 & 57.66 & 68.67 & 69.33 & 0.60 & 0.80 & 0.90 & 0.60 & 0.80 & 0.90 \\
\hline 34 & 30.33 & 70.33 & 60.67 & 45.00 & 68.66 & 60.67 & 0.44 & 0.70 & 0.92 & 0.33 & 0.70 & 0.92 \\
\hline 35 & 48.00 & 59.33 & 56.00 & 56.66 & 63.00 & 69.66 & 0.40 & 0.80 & 0.94 & 0.40 & 0.80 & 0.94 \\
\hline 36 & 32.33 & 31.67 & 52.33 & 44.66 & 45.68 & 55.33 & 0.40 & 0.80 & 0.92 & 0.40 & 0.80 & 0.92 \\
\hline 37 & 54.00 & 79.33 & 89.33 & 55.66 & 75.70 & 89.33 & 0.30 & 0.70 & 0.80 & 0.40 & 0.70 & 0.80 \\
\hline 38 & 50.00 & 60.00 & 92.00 & 50.00 & 60.66 & 92.00 & 0.50 & 0.80 & 0.80 & 0.50 & 0.80 & 0.83 \\
\hline 39 & 40.00 & 60.00 & 90.00 & 42.00 & 57.70 & 90.00 & 0.60 & 0.80 & 0.88 & 0.60 & 0.80 & 0.88 \\
\hline 40 & 60.00 & 70.00 & 98.00 & 62.00 & 70.00 & 95.33 & 0.65 & 0.90 & 0.96 & 0.75 & 0.86 & 0.98 \\
\hline LSD $5 \%$ & 2.412 & 3.368 & 2.941 & 2.607 & 3.166 & 3.079 & 0.043 & 0.041 & 0.043 & 0.038 & 0.041 & 0.041 \\
\hline LSD $1 \%$ & 3.469 & 4.846 & 4.231 & 3.752 & 4.556 & 4.430 & 0.062 & 0.059 & 0.062 & 0.055 & 0.059 & 0.059 \\
\hline
\end{tabular}


TABLE 6. The mean performance of the studied rice accessions for three sowing dates at two years of 2015 and 2016 for seedling height and root length

\begin{tabular}{|c|c|c|c|c|c|c|c|c|c|c|c|c|}
\hline \multirow{3}{*}{ Genotypes } & \multicolumn{6}{|c|}{ Seedling height $(\mathrm{cm})$} & \multicolumn{6}{|c|}{ Root length $(\mathrm{cm})$} \\
\hline & \multicolumn{3}{|c|}{2015} & \multicolumn{3}{|c|}{2016} & \multicolumn{3}{|c|}{2015} & \multicolumn{3}{|c|}{2016} \\
\hline & $1^{\text {st }}$ March & $\begin{array}{c}1^{\text {st }} \\
\text { April }\end{array}$ & $\begin{array}{c}1^{\text {st }} \\
\text { May }\end{array}$ & $1^{\text {st }}$ March & $\begin{array}{c}1^{\text {st }} \\
\text { April }\end{array}$ & $\begin{array}{c}1^{\text {st }} \\
\text { May }\end{array}$ & $1^{\text {st }}$ March & $\begin{array}{c}1^{\text {st }} \\
\text { April }\end{array}$ & $\begin{array}{c}1^{\text {st }} \\
\text { May }\end{array}$ & $1^{\text {st }}$ March & $\begin{array}{c}1^{\mathrm{st}} \\
\text { April }\end{array}$ & $\begin{array}{c}1^{\text {st }} \\
\text { May }\end{array}$ \\
\hline 1 & 4.00 & 5.00 & 7.67 & 4.00 & 4.87 & 7.33 & 3.50 & 4.17 & 4.67 & 3.50 & 4.66 & 5.66 \\
\hline 2 & 2.83 & 4.00 & 5.50 & 4.83 & 4.10 & 5.57 & 2.67 & 4.33 & 4.33 & 3.50 & 4.33 & 4.66 \\
\hline 3 & 2.83 & 3.83 & 5.83 & 3.17 & 3.83 & 5.83 & 2.33 & 4.83 & 5.33 & 3.00 & 4.66 & 5.33 \\
\hline 4 & 2.83 & 5.17 & 7.00 & 3.33 & 5.23 & 7.00 & 2.50 & 5.00 & 5.50 & 2.33 & 5.00 & 5.50 \\
\hline 5 & 3.17 & 4.67 & 6.83 & 2.67 & 4.50 & 6.83 & 2.83 & 4.67 & 6.00 & 2.00 & 4.67 & 6.00 \\
\hline 6 & 2.83 & 4.33 & 7.00 & 2.67 & 4.33 & 7.50 & 2.33 & 3.83 & 6.33 & 2.00 & 3.66 & 6.33 \\
\hline 7 & 1.83 & 4.67 & 7.67 & 3.17 & 4.50 & 7.67 & 2.50 & 3.67 & 5.83 & 2.67 & 3.67 & 5.83 \\
\hline 8 & 3.00 & 4.67 & 6.50 & 3.33 & 4.67 & 6.57 & 3.00 & 3.83 & 5.00 & 3.00 & 3.66 & 5.00 \\
\hline 9 & 3.67 & 4.33 & 6.83 & 2.67 & 4.30 & 6.83 & 3.17 & 4.17 & 5.17 & 1.83 & 4.33 & 5.33 \\
\hline 10 & 3.67 & 5.33 & 6.83 & 2.50 & 5.40 & 6.83 & 2.83 & 3.50 & 5.67 & 2.00 & 3.50 & 5.67 \\
\hline 11 & 3.50 & 4.17 & 5.50 & 2.67 & 4.00 & 5.67 & 3.33 & 3.83 & 4.83 & 2.83 & 3.83 & 4.83 \\
\hline 12 & 3.67 & 5.33 & 7.67 & 3.83 & 5.50 & 7.67 & 3.17 & 3.83 & 6.00 & 3.33 & 3.60 & 6.00 \\
\hline 13 & 3.00 & 4.33 & 7.67 & 3.17 & 4.50 & 7.67 & 3.00 & 3.50 & 5.50 & 3.17 & 3.50 & 5.50 \\
\hline 14 & 4.50 & 4.50 & 9.00 & 3.50 & 4.67 & 8.50 & 3.00 & 4.33 & 6.17 & 2.67 & 4.33 & 6.33 \\
\hline 15 & 2.83 & 5.50 & 9.67 & 2.50 & 5.33 & 8.67 & 2.83 & 3.33 & 6.00 & 2.33 & 3.33 & 6.00 \\
\hline 16 & 3.67 & 4.17 & 7.67 & 3.67 & 4.33 & 7.67 & 3.17 & 3.17 & 5.00 & 3.17 & 3.00 & 5.00 \\
\hline 17 & 3.33 & 5.33 & 9.67 & 3.67 & 5.33 & 8.67 & 3.17 & 3.67 & 7.30 & 3.33 & 3.67 & 7.20 \\
\hline 18 & 2.83 & 4.00 & 7.67 & 3.67 & 4.00 & 7.67 & 2.67 & 3.33 & 4.50 & 3.00 & 3.33 & 4.50 \\
\hline 19 & 3.67 & 5.83 & 8.33 & 3.83 & 5.67 & 8.33 & 3.33 & 4.33 & 6.83 & 3.50 & 4.33 & 6.83 \\
\hline 20 & 3.67 & 4.33 & 9.33 & 2.50 & 4.67 & 8.33 & 3.00 & 3.67 & 6.67 & 2.50 & 3.67 & 6.33 \\
\hline 21 & 2.00 & 4.33 & 8.50 & 2.67 & 4.33 & 8.50 & 2.17 & 2.33 & 7.00 & 2.33 & 3.00 & 7.00 \\
\hline 22 & 2.50 & 4.50 & 7.00 & 2.67 & 4.33 & 7.00 & 2.50 & 2.50 & 6.67 & 2.17 & 2.66 & 6.67 \\
\hline 23 & 2.73 & 4.33 & 7.33 & 2.83 & 4.33 & 7.33 & 2.17 & 2.50 & 5.50 & 2.33 & 2.33 & 5.50 \\
\hline 24 & 3.50 & 5.00 & 9.00 & 3.83 & 5.33 & 8.00 & 2.67 & 3.17 & 7.00 & 2.33 & 3.17 & 7.33 \\
\hline 25 & 3.00 & 5.33 & 7.00 & 2.83 & 4.67 & 6.83 & 2.83 & 3.33 & 6.67 & 2.17 & 3.67 & 6.17 \\
\hline 26 & 3.33 & 4.50 & 8.33 & 2.67 & 4.67 & 8.33 & 2.50 & 3.83 & 7.17 & 2.33 & 3.67 & 7.00 \\
\hline 27 & 3.33 & 5.33 & 7.83 & 2.33 & 5.33 & 7.67 & 3.33 & 4.17 & 5.83 & 2.17 & 4.00 & 5.83 \\
\hline 28 & 3.00 & 4.83 & 8.67 & 2.33 & 5.33 & 8.33 & 2.67 & 3.27 & 6.50 & 2.17 & 3.00 & 6.33 \\
\hline 29 & 1.83 & 4.67 & 8.00 & 2.17 & 4.67 & 8.00 & 1.83 & 2.83 & 6.33 & 2.00 & 3.00 & 6.33 \\
\hline 30 & 2.50 & 4.33 & 6.00 & 2.33 & 4.33 & 6.00 & 2.67 & 3.33 & 5.00 & 2.50 & 3.33 & 5.00 \\
\hline 31 & 2.50 & 3.83 & 6.00 & 2.33 & 3.67 & 6.00 & 2.50 & 2.50 & 4.33 & 2.50 & 2.50 & 4.33 \\
\hline 32 & 2.50 & 3.33 & 6.33 & 1.67 & 3.67 & 6.33 & 2.67 & 3.50 & 5.17 & 2.66 & 3.50 & 5.33 \\
\hline 33 & 2.50 & 5.17 & 7.00 & 2.17 & 5.33 & 7.00 & 2.67 & 3.17 & 6.17 & 2.33 & 3.17 & 6.17 \\
\hline 34 & 2.50 & 5.50 & 7.33 & 2.50 & 5.50 & 7.33 & 2.67 & 3.83 & 5.17 & 1.83 & 3.83 & 5.33 \\
\hline 35 & 2.33 & 4.50 & 6.67 & 2.67 & 4.50 & 6.67 & 2.17 & 2.33 & 5.17 & 2.33 & 2.66 & 5.17 \\
\hline 36 & 2.17 & 3.00 & 5.50 & 2.33 & 3.00 & 5.50 & 2.00 & 2.00 & 3.83 & 2.00 & 2.66 & 4.00 \\
\hline 37 & 2.83 & 4.17 & 7.00 & 2.83 & 4.17 & 7.00 & 2.50 & 2.17 & 4.67 & 2.50 & 2.33 & 4.67 \\
\hline 38 & 2.33 & 3.83 & 6.00 & 3.33 & 4.00 & 6.00 & 2.67 & 4.00 & 5.00 & 3.00 & 3.00 & 5.00 \\
\hline 39 & 3.00 & 4.00 & 6.00 & 3.33 & 4.00 & 6.33 & 4.00 & 4.00 & 6.33 & 3.00 & 4.00 & 6.00 \\
\hline 40 & 5.00 & 8.00 & 9.00 & 4.00 & 7.67 & 8.67 & 5.00 & 6.00 & 8.00 & 4.33 & 6.66 & 8.33 \\
\hline LSD $5 \%$ & 0.234 & 0.307 & 0.445 & 0.242 & 0.355 & 0.417 & 0.304 & 0.350 & 0.407 & 0.339 & 0.330 & 0.393 \\
\hline LSD $1 \%$ & 0.336 & 0.442 & 0.639 & 0.346 & 0.468 & 0.598 & 0.436 & 0.504 & 0.586 & 0.488 & 0.476 & 0.566 \\
\hline
\end{tabular}

J. Sus. Agric .Sci. 44, No.2 (2018) 
TABLE 7. The mean performance of the studied rice accessions for three sowing dates at two years of 2015 and 2016 for number of leaves/seedling

\begin{tabular}{|c|c|c|c|c|c|c|}
\hline \multirow{3}{*}{ Genotypes } & \multicolumn{6}{|c|}{ No. of leaves/seedling } \\
\hline & \multicolumn{3}{|c|}{2015} & \multicolumn{3}{|c|}{2016} \\
\hline & $1^{\text {st }}$ March & $\mathbf{1}^{\text {st }}$ April & $1^{\text {st }}$ May & $1^{\text {st }}$ March & $1^{\text {st }}$ April & $1^{\text {st }}$ May \\
\hline 1 & 2.66 & 3.00 & 3.66 & 3.00 & 3.00 & 3.66 \\
\hline 2 & 2.66 & 2.00 & 4.66 & 2.66 & 2.00 & 4.00 \\
\hline 3 & 1.33 & 1.66 & 3.00 & 1.33 & 1.66 & 2.66 \\
\hline 4 & 1.33 & 2.00 & 2.00 & 1.66 & 2.00 & 2.00 \\
\hline 5 & 2.00 & 2.33 & 3.66 & 2.00 & 2.33 & 3.66 \\
\hline 6 & 2.00 & 2.00 & 2.66 & 2.00 & 2.00 & 2.66 \\
\hline 7 & 1.66 & 2.00 & 2.66 & 1.66 & 2.00 & 2.66 \\
\hline 8 & 2.00 & 2.00 & 3.66 & 2.00 & 2.00 & 3.66 \\
\hline 9 & 3.66 & 3.66 & 4.00 & 3.66 & 3.66 & 4.33 \\
\hline 10 & 1.33 & 1.00 & 2.66 & 1.33 & 1.00 & 3.00 \\
\hline 11 & 1.33 & 2.66 & 3.00 & 1.66 & 2.33 & 3.00 \\
\hline 12 & 3.00 & 3.66 & 4.00 & 3.00 & 3.66 & 4.00 \\
\hline 13 & 1.00 & 1.00 & 2.33 & 1.00 & 1.33 & 2.66 \\
\hline 14 & 1.00 & 1.00 & 2.66 & 1.00 & 1.00 & 2.66 \\
\hline 15 & 2.66 & 1.00 & 4.00 & 2.66 & 1.00 & 4.00 \\
\hline 16 & 1.33 & 1.66 & 3.00 & 1.00 & 1.66 & 2.33 \\
\hline 17 & 1.66 & 1.00 & 2.33 & 1.66 & 1.66 & 3.00 \\
\hline 18 & 1.00 & 1.66 & 3.00 & 1.00 & 1.66 & 2.66 \\
\hline 19 & 2.66 & 3.00 & 4.00 & 2.66 & 3.33 & 4.00 \\
\hline 20 & 1.66 & 2.00 & 3.00 & 1.66 & 2.00 & 3.00 \\
\hline 21 & 1.66 & 1.00 & 2.66 & 1.66 & 1.66 & 3.00 \\
\hline 22 & 1.00 & 1.33 & 2.00 & 1.00 & 1.00 & 2.33 \\
\hline 23 & 1.00 & 1.00 & 2.33 & 1.00 & 1.00 & 2.66 \\
\hline 24 & 2.66 & 3.00 & 4.00 & 2.66 & 3.33 & 3.66 \\
\hline 25 & 1.66 & 2.33 & 3.66 & 1.66 & 2.2 .5 & 4.00 \\
\hline 26 & 3.00 & 3.33 & 4.00 & 3.33 & 3.00 & 4.33 \\
\hline 27 & 1.33 & 2.66 & 3.66 & 1.33 & 2.33 & 3.66 \\
\hline 28 & 1.33 & 2.66 & 4.00 & 1.33 & 2.00 & 4.00 \\
\hline 29 & 1.33 & 2.00 & 4.33 & 1.33 & 2.33 & 4.33 \\
\hline 30 & 1.00 & 1.00 & 2.00 & 1.00 & 1.00 & 2.33 \\
\hline 31 & 1.00 & 1.33 & 3.33 & 1.00 & 1.00 & 3.33 \\
\hline 32 & 1.00 & 1.00 & 2.00 & 1.00 & 1.00 & 2.00 \\
\hline 33 & 1.00 & 1.66 & 3.66 & 1.00 & 1.66 & 3.66 \\
\hline 34 & 1.00 & 1.66 & 2.00 & 1.00 & 1.66 & 2.33 \\
\hline 35 & 1.00 & 1.66 & 2.00 & 1.00 & 1.66 & 2.33 \\
\hline 36 & 1.00 & 1.00 & 1.66 & 1.00 & 1.00 & 1.66 \\
\hline 37 & 1.00 & 1.00 & 1.66 & 1.00 & 1.00 & 1.66 \\
\hline 38 & 2.00 & 2.00 & 3.00 & 2.00 & 2.00 & 3.00 \\
\hline 39 & 2.33 & 2.00 & 4.00 & 2.33 & 2.00 & 4.00 \\
\hline 40 & 2.33 & 3.33 & 4.00 & 2.66 & 3.33 & 4.33 \\
\hline LSD $5 \%$ & 0.339 & 0.381 & 0.345 & 0.313 & 0.361 & 0.371 \\
\hline LSD $1 \%$ & 0.488 & 0.548 & 0.496 & 0.450 & 0.518 & 0.534 \\
\hline
\end{tabular}


TABLE 8. Selected accessions for cold tolerance based on cold reduction index (\%) using combined data over all two growing seasons

\begin{tabular}{|c|c|c|c|c|c|c|}
\hline NO & Genotypes & $\begin{array}{c}\text { Germination } \\
\text { percentage }\end{array}$ & $\begin{array}{c}\text { Germination } \\
\text { index }\end{array}$ & $\begin{array}{c}\text { Seedling } \\
\text { height }\end{array}$ & $\begin{array}{l}\text { Root } \\
\text { length }\end{array}$ & $\begin{array}{c}\text { no. of leaves } \\
\text { /seedling }\end{array}$ \\
\hline 1 & CT6749-36-7-2-M-M (1) & 57.97 & 32.65 & 45.43 & 38.16 & 22.68 \\
\hline 2 & H270-30-2-1 (2) & 82.85 & 36.17 & 31.24 & 33.80 & 33.50 \\
\hline 3 & Milyang 80 (9) & 49.20 & 16.67 & 53.59 & 53.10 & 15.47 \\
\hline 4 & PSRM1-17-4B-13 (11) & 60.18 & 33.33 & 45.59 & 36.23 & 44.67 \\
\hline 5 & Ryong Sung 25 (12) & 49.66 & 25.79 & 51.11 & 45.83 & 25.00 \\
\hline 6 & Giza 177 (19) & 13.07 & 23.20 & 54.98 & 50.00 & 33.50 \\
\hline 7 & Sakha102 (24) & 22.28 & 25.00 & 59.28 & 65.89 & 27.32 \\
\hline 8 & Sakha 104 (26) & 11.82 & 25.51 & 63.99 & 65.50 & 26.91 \\
\hline 9 & СТ9882-16-4-2-3-2-p-M (39) & 54.44 & 31.82 & 50.00 & 41.67 & 41.75 \\
\hline
\end{tabular}

\section{Cluster based on morphological dendrogram}

Cluster Analysis was used to arrange a set of variables into clusters. Its objective was to sort cases (variables) into groups, or clusters, so the degree of association was strong within members of the same cluster and weak between members of different clusters. This description maybe abstracted through use of the particular to the general class or type (Anderberg, 1973).

The cluster analysis for the data obtained from phenotyping, (Figure 5) showed that the phylogenetic analysis divided the studied accessions into two main groups (A and B) in addition to some sub groups belong to each main group. Group A including 13 rice accessions with the majority of Indica and Indica/ Japonica types and a few numbers of Japonica type accessions only three. In general, the group (A) including the rice accessions that showed poor or moderate performance under low temperature conditions. On other hand the group B including 27 rice accessions divided into two sub groups, the majority of these sub groups for Japonica accessions with 13 accessions, six Indica/ Japonica accessions and eight Indica type. Evidently, the group B including the most superior rice accessions under low temperature conditions. these results are in agreement with the previous results which reported that Japonica type accessions are more cold tolerance than Indica type (Renata et al., 2010; Sanghera et al., 2011 and Suh et al., 2013). Due to the useful breeding programs are depending on modern and adapted knowledge of genetic diversity among varieties to utilize the available genetic resources to create new genotypes, morphological markers reflect not only the genetic contribution of the genotypes but also the interaction of the genotype with environment in which it is revealed (Thenmozhi and Rajasekaran, 2013).

\section{Genotyping}

\section{SSR polymorphism}

The objective of this investigation to evaluate the ability of cold tolerance for the 40 rice accessions based on their genetic diversity using molecular markers related with cold tolerance at seedling stage. Out of 36 SSR markers used in this study, four markers showed highly polymorphic pattern among the studied genotypes, the electrophotogram for the amplified DNA fragments for these markers are shown in Fig. 1- 4. The number of polymorphic alleles ranged from five by RM324 to nine by RM265 markers. The RM265 marker amplified nine different alleles for the studied rice accessions (Figure 1), the molecular weight of these alleles ranged between $460 \mathrm{bp}$ and $160 \mathrm{bp}$, while the RM324 marker amplified five different alleles, (figure 2), the molecular weight of these alleles ranged between 290 bp and 120 bp. The RM163 marker amplified nine different alleles with molecular weight ranged between $490 \mathrm{bp}$ and $230 \mathrm{bp}$ (Fig. 3). The RM270 marker amplified eight different alleles with molecular weight ranged between $250 \mathrm{bp}$ and $100 \mathrm{bp}$ (Fig. 4).

\section{Phylogenetic analysis}

Regarding the cluster analysis for the data obtained from the four SSR markers (Figure 6) the results showed that the phylogenetic analysis divided the studied accessions into two main groups (A and $\mathrm{B}$ ) in addition to some sub groups belong each main group. Group A included 18 rice accessions with the majority of Indica and Indica/ Japonica types and a few number of Japonica type accessions only four accessions, in general the results Indicated that this 
group (A) including accessions with poor or moderate ability for cold tolerance under low temperature conditions. On other hand the group B included 22 accessions divided into two sub groups, the majority of these sub groups for Japonica accessions with 11 accessions, five Indica/ Japonica accessions and six Indica type. Apparently, the group B including the most of superior accessions under low temperature conditions such as CT6749-36-7-2-M-M, Milyang 80, PSRM1-17-4B-13, Ryong Sung 25, Giza 177, Sakha102, Sakha 104, CT9882-16-4-2-3-2-p-M, these results confirming the phenotypic results. These results are in a harmony with those reported by Suh et al., 2013.
Molecular markers have the potential to determine genetic diversity and to help the management of plant genetic resources. In contrary of morphological traits, molecular markers can demonstrate the differences among genotypes at the DNA level, providing a more direct, accurate and efficient tool for germplasm characterization and management. This emphasizes the urgent need of molecular mapping for cold tolerance to detect linked DNA marker(s). Doing so will greatly help in improving selection efficiency for cold tolerance.

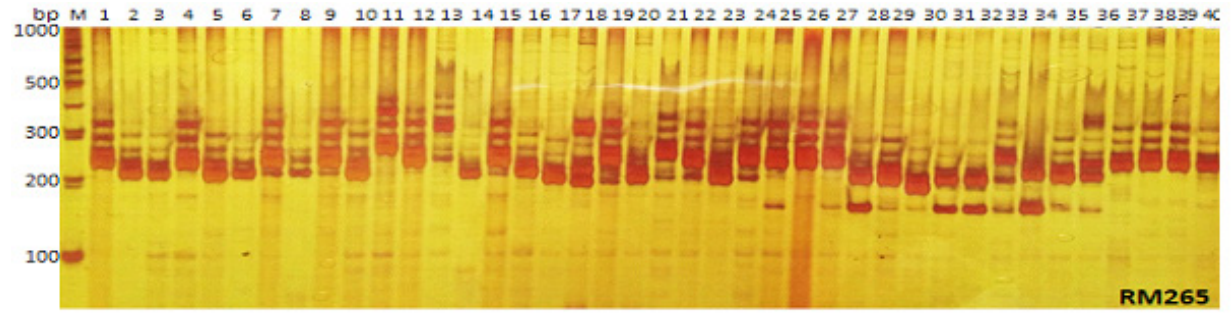

Fig. 1. Genetic Polymorphism among the 40 genotypes using RM 265

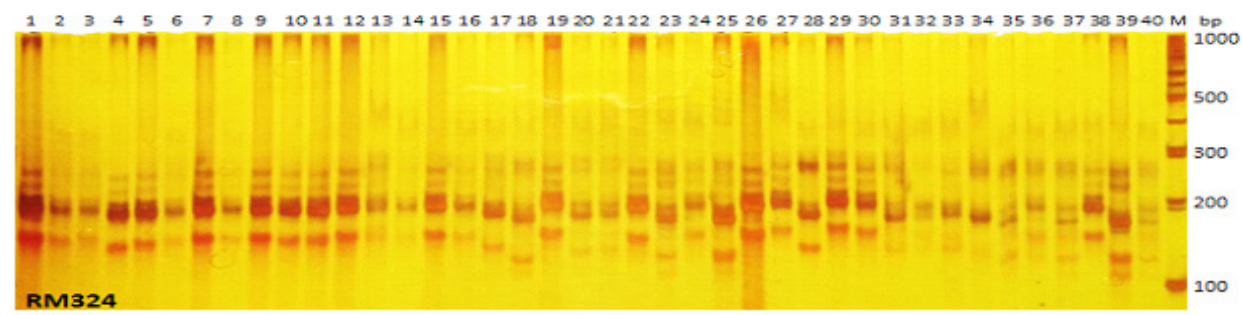

Fig. 2. Genetic Polymorphism among the 40 genotypes using RM 324

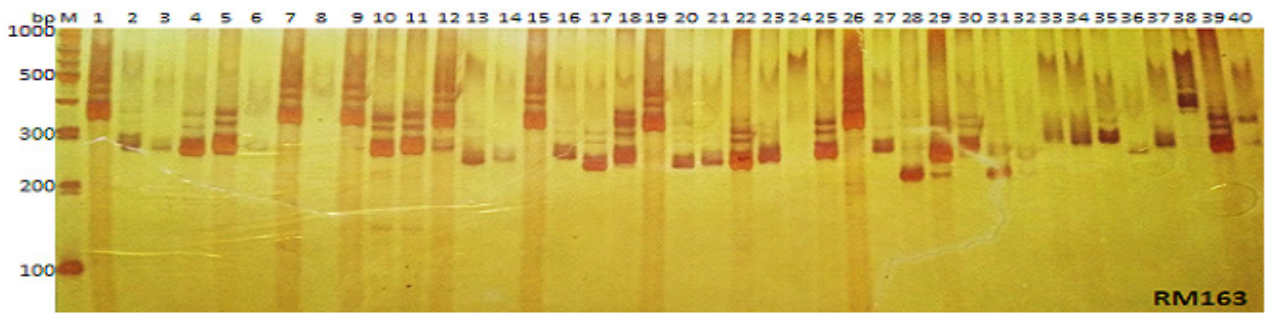

Fig. 3. Genetic Polymorphism among the 40 genotypes using RM 163

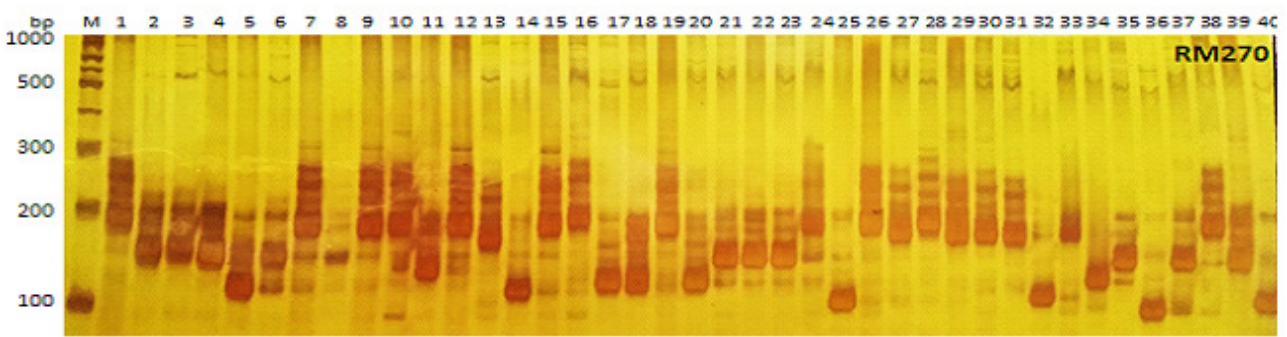

Fig. 4. Genetic Polymorphism among the 40 genotypes using RM 270 


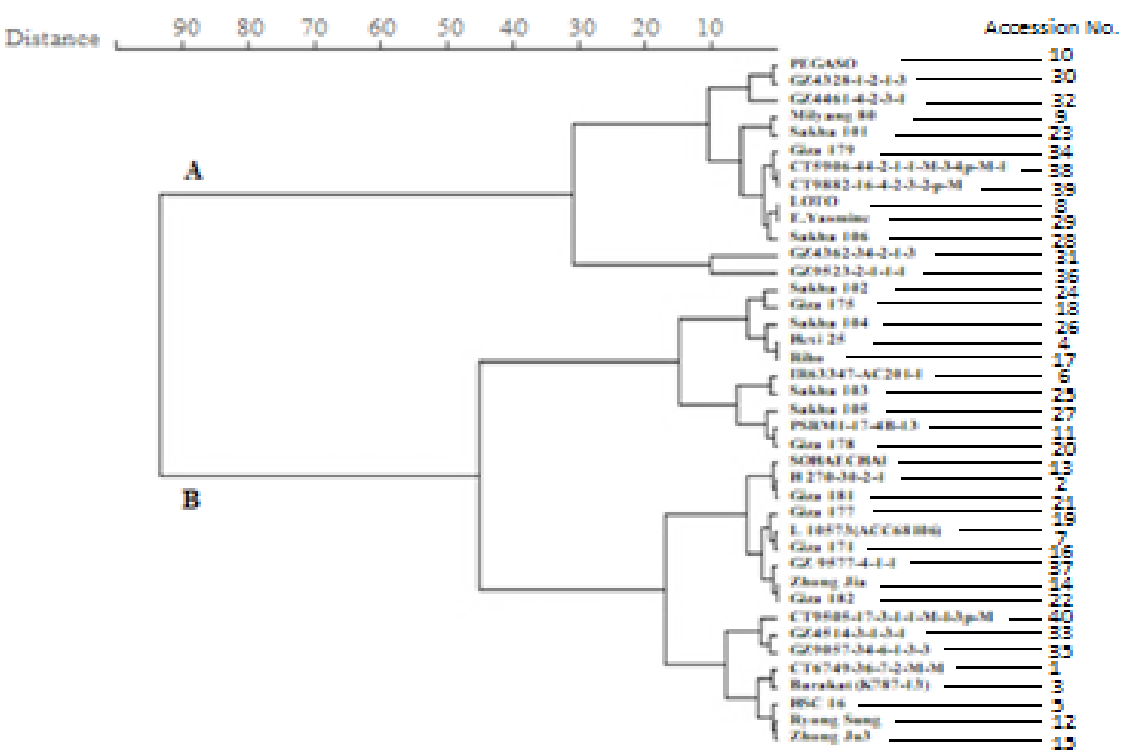

Fig. 5. Dendrogram of 40 rice accessions based on phenotyping data using hierarchical cluster analysis (wards method)

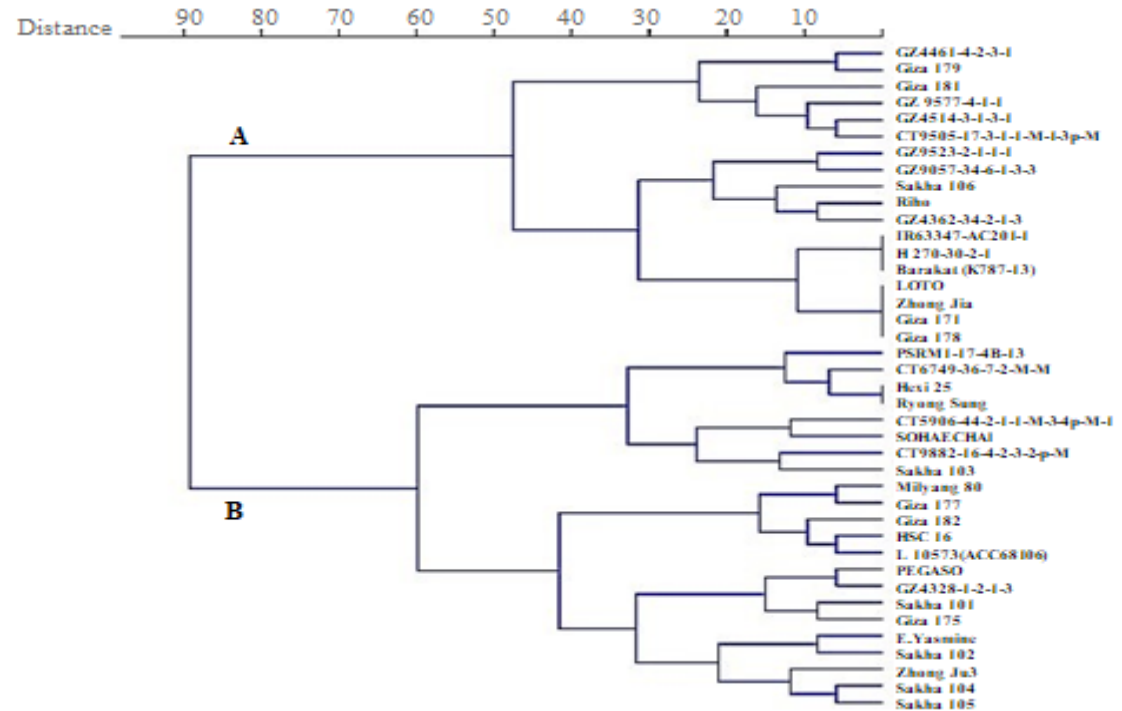

Fig. 6. Dendrogram of 40 rice accessions for cold tolerance, clustered using four SSR markers related with cold tolerance at seedling stage

TABLE 9. Matrix for SSR amplified fragments for the studied genotypes at the locus RM265

MW $11220 \begin{array}{llllllllllllllllllllllllllllllllllllll} & 5 & 6 & 7 & 8 & 9 & 10 & 11 & 12 & 13 & 14 & 15 & 16 & 17 & 18 & 19 & 20 & 21 & 22 & 23 & 24 & 25 & 26 & 27 & 28 & 29 & 30 & 31 & 32 & 33 & 34 & 35 & 36 & 37 & 38 & 39 & 40\end{array}$

$400 \begin{array}{lllllllllllllllllllllllllllllllllllllllllllllll} & 0 & 0 & 0 & 0 & 0 & 0 & 0 & 0 & 0 & 0 & 1 & 1 & 1 & 0 & 0 & 0 & 0 & 0 & 0 & 0 & 0 & 0 & 0 & 0 & 0 & 1 & 0 & 0 & 0 & 0 & 0 & 0 & 0 & 0 & 0 & 0 & 0 & 0 & 0 & 0\end{array}$ $\begin{array}{llllllllllllllllllllllllllllllllllllllllllllllll}340 & 1 & 0 & 0 & 0 & 0 & 0 & 1 & 0 & 1 & 0 & 1 & 1 & 1 & 0 & 1 & 0 & 0 & 1 & 1 & 0 & 1 & 1 & 0 & 1 & 1 & 1 & 1 & 0 & 0 & 0 & 0 & 0 & 0 & 0 & 0 & 1 & 1 & 1 & 1 & 0\end{array}$ $\begin{array}{llllllllllllllllllllllllllllllllllllllllllll}300 & 1 & 1 & 1 & 1 & 1 & 0 & 1 & 0 & 1 & 1 & 1 & 1 & 0 & 0 & 1 & 0 & 0 & 0 & 1 & 0 & 0 & 1 & 0 & 1 & 0 & 1 & 1 & 0 & 0 & 0 & 0 & 0 & 0 & 0 & 1 & 0 & 1 & 1 & 1 & 0\end{array}$ $\begin{array}{llllllllllllllllllllllllllllllllllllllllllllllllllll}270 & 0 & 1 & 1 & 1 & 1 & 0 & 0 & 0 & 1 & 1 & 1 & 1 & 0 & 0 & 0 & 1 & 0 & 0 & 0 & 0 & 1 & 1 & 0 & 1 & 1 & 1 & 1 & 0 & 1 & 0 & 0 & 0 & 1 & 0 & 1 & 0 & 1 & 1 & 1 & 0\end{array}$ $\begin{array}{llllllllllllllllllllllllllllllllllllllllllllll}240 & 1 & 0 & 0 & 1 & 0 & 1 & 1 & 1 & 1 & 1 & 0 & 1 & 1 & 0 & 1 & 1 & 0 & 0 & 1 & 1 & 1 & 1 & 1 & 1 & 1 & 1 & 1 & 1 & 1 & 0 & 0 & 0 & 0 & 1 & 0 & 1 & 1 & 1 & 1 & 1\end{array}$ $\begin{array}{llllllllllllllllllllllllllllllllllllllllllllllll}215 & 0 & 1 & 1 & 0 & 1 & 1 & 1 & 1 & 0 & 1 & 0 & 0 & 0 & 1 & 0 & 1 & 1 & 1 & 1 & 1 & 0 & 0 & 1 & 1 & 0 & 0 & 0 & 0 & 1 & 0 & 1 & 1 & 1 & 1 & 1 & 1 & 0 & 0 & 0 & 0\end{array}$ $200 \begin{array}{lllllllllllllllllllllllllllllllllllllllllllll}20 & 0 & 0 & 0 & 0 & 0 & 0 & 0 & 0 & 0 & 0 & 0 & 0 & 0 & 0 & 0 & 1 & 0 & 0 & 0 & 0 & 1 & 0 & 0 & 0 & 0 & 1 & 1 & 0 & 1 & 1 & 0 & 0 & 1 & 0 & 0 & 0 & 0 & 0\end{array}$ $\begin{array}{lllllllllllllllllllllllllllllllllllllllllllll}190 & 0 & 0 & 0 & 0 & 0 & 0 & 0 & 0 & 0 & 0 & 0 & 0 & 0 & 0 & 0 & 0 & 0 & 0 & 0 & 0 & 0 & 0 & 0 & 0 & 0 & 0 & 0 & 0 & 0 & 1 & 0 & 0 & 0 & 0 & 0 & 0 & 0 & 0 & 0 & 0\end{array}$ $160 \begin{array}{llllllllllllllllllllllllllllllllllllllllllllllllllll} & 0 & 0 & 0 & 0 & 0 & 0 & 0 & 0 & 0 & 0 & 0 & 0 & 0 & 0 & 0 & 0 & 0 & 0 & 0 & 0 & 0 & 0 & 0 & 1 & 0 & 0 & 1 & 0 & 0 & 1 & 1 & 1 & 1 & 0 & 0 & 0 & 0 & 0 & 0\end{array}$ J. Sus. Agric .Sci. 44, No.2 (2018) 
TABLE 10. Matrix for SSR amplified fragments for the studied genotypes at the locus RM324

MW $112 \begin{array}{lllllllllllllllllllllllllllllllllllllll} & 3 & 4 & 5 & 6 & 7 & 8 & 9 & 10 & 11 & 12 & 13 & 14 & 15 & 16 & 17 & 18 & 19 & 20 & 21 & 22 & 23 & 24 & 25 & 26 & 27 & 28 & 29 & 30 & 31 & 32 & 33 & 34 & 35 & 36 & 37 & 38 & 39 & 40\end{array}$

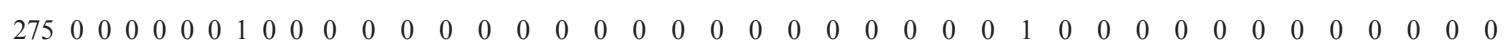
$\begin{array}{llllllllllllllllllllllllllllllllllllllllllllllll}200 & 1 & 1 & 1 & 1 & 1 & 1 & 1 & 1 & 1 & 1 & 1 & 1 & 0 & 0 & 1 & 0 & 0 & 0 & 1 & 0 & 0 & 1 & 0 & 0 & 0 & 1 & 1 & 0 & 1 & 1 & 0 & 0 & 0 & 0 & 0 & 0 & 0 & 1 & 0 & 0\end{array}$ $190 \begin{array}{llllllllllllllllllllllllllllllllllllllllllll} & 0 & 0 & 0 & 0 & 0 & 0 & 0 & 0 & 0 & 0 & 0 & 0 & 0 & 0 & 0 & 0 & 1 & 0 & 0 & 0 & 0 & 1 & 0 & 1 & 0 & 0 & 0 & 0 & 0 & 0 & 0 & 0 & 1 & 0 & 0 & 0 & 0 & 1 & 0\end{array}$ $\begin{array}{llllllllllllllllllllllllllllllllllllllllllllll}165 & 1 & 1 & 0 & 1 & 1 & 1 & 1 & 1 & 1 & 0 & 0 & 1 & 0 & 0 & 0 & 1 & 0 & 0 & 1 & 0 & 0 & 0 & 1 & 0 & 0 & 1 & 1 & 0 & 0 & 0 & 0 & 0 & 0 & 0 & 1 & 0 & 0\end{array}$ $150 \begin{array}{lllllllllllllllllllllllllllllllllllllllllllll} & 0 & 0 & 1 & 0 & 0 & 0 & 0 & 0 & 0 & 0 & 0 & 0 & 0 & 0 & 0 & 0 & 0 & 0 & 0 & 0 & 0 & 0 & 0 & 0 & 0 & 1 & 0 & 0 & 0 & 0 & 0 & 0 & 0 & 0 & 0 & 0 & 0 & 0 & 0\end{array}$ $140 \begin{array}{lllllllllllllllllllllllllllllllllllllllllllll} & 0 & 0 & 1 & 0 & 0 & 0 & 0 & 0 & 0 & 0 & 0 & 0 & 0 & 0 & 0 & 0 & 0 & 0 & 0 & 0 & 0 & 0 & 1 & 0 & 0 & 0 & 0 & 0 & 0 & 0 & 0 & 0 & 0 & 0 & 0 & 0 & 0 & 1 & 0\end{array}$

TABLE 11. Matrix for SSR amplified fragments for the studied genotypes at the locus RM163

MW $1 \begin{array}{llllllllllllllllllllllllllllllllllllllll} & 2 & 3 & 4 & 5 & 6 & 7 & 8 & 9 & 10 & 11 & 12 & 13 & 14 & 15 & 16 & 17 & 18 & 19 & 20 & 21 & 22 & 23 & 24 & 25 & 26 & 27 & 28 & 29 & 30 & 31 & 32 & 33 & 34 & 35 & 36 & 37 & 38 & 39 & 40\end{array}$ $\begin{array}{llllllllllllllllllllllllllllllllllllllllll}410 & 1 & 0 & 0 & 0 & 0 & 0 & 1 & 0 & 1 & 0 & 0 & 1 & 0 & 0 & 1 & 0 & 0 & 0 & 1 & 0 & 0 & 0 & 0 & 0 & 0 & 1 & 0 & 0 & 0 & 0 & 0 & 0 & 0 & 0 & 0 & 0 & 0 & 1 & 0 & 0\end{array}$ $\begin{array}{llllllllllllllllllllllllllllllllllllllllllllllll}380 & 1 & 0 & 0 & 0 & 0 & 0 & 0 & 0 & 0 & 0 & 0 & 0 & 0 & 0 & 1 & 0 & 0 & 0 & 1 & 0 & 0 & 0 & 0 & 0 & 0 & 1 & 0 & 0 & 0 & 0 & 0 & 0 & 0 & 0 & 0 & 0 & 0 & 1 & 0 & 0\end{array}$ $\begin{array}{lllllllllllllllllllllllllllllllllllllllllllll}330 & 1 & 0 & 0 & 1 & 1 & 1 & 1 & 0 & 1 & 0 & 1 & 0 & 0 & 0 & 0 & 0 & 0 & 1 & 0 & 0 & 0 & 0 & 0 & 0 & 0 & 1 & 0 & 0 & 0 & 0 & 0 & 0 & 0 & 0 & 0 & 0 & 0 & 0 & 1 & 1\end{array}$

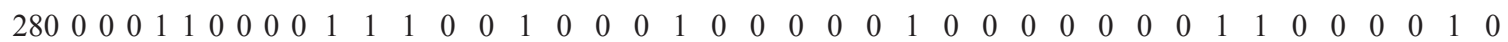
$\begin{array}{llllllllllllllllllllllllllllllllllllllllllllllll}250 & 0 & 1 & 1 & 1 & 1 & 0 & 0 & 0 & 0 & 1 & 1 & 1 & 0 & 0 & 0 & 1 & 0 & 0 & 0 & 0 & 0 & 0 & 0 & 0 & 0 & 0 & 0 & 0 & 0 & 0 & 0 & 0 & 1 & 1 & 1 & 0 & 0 & 0 & 0 & 0\end{array}$

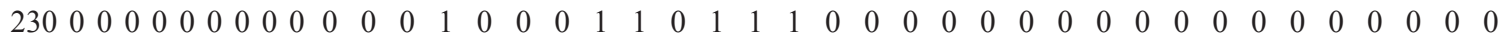

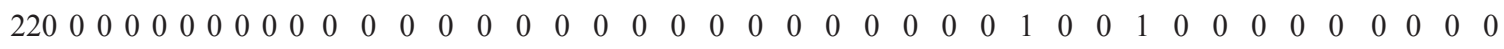

TABLE 12. Matrix for SSR amplified fragments for the studied genotypes at the locus RM270

$\begin{array}{lllllllllllllllllllllllllllllllllllllllll}\text { MW } & 1 & 2 & 3 & 4 & 5 & 6 & 7 & 8 & 9 & 10 & 11 & 12 & 13 & 14 & 15 & 16 & 17 & 18 & 19 & 20 & 21 & 22 & 23 & 24 & 25 & 26 & 27 & 28 & 29 & 30 & 31 & 32 & 33 & 34 & 35 & 36 & 37 & 38 & 39 & 40\end{array}$

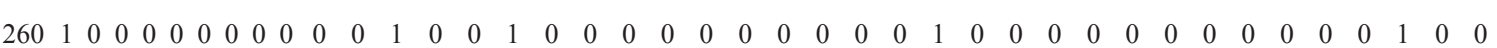
$230 \begin{array}{lllllllllllllllllllllllllllllllllllllllllllll} & 0 & 0 & 0 & 0 & 0 & 1 & 0 & 1 & 1 & 0 & 1 & 1 & 0 & 1 & 1 & 0 & 0 & 1 & 0 & 0 & 0 & 0 & 0 & 0 & 1 & 1 & 1 & 0 & 0 & 0 & 0 & 0 & 0 & 0 & 0 & 0 & 1 & 0 & 0\end{array}$

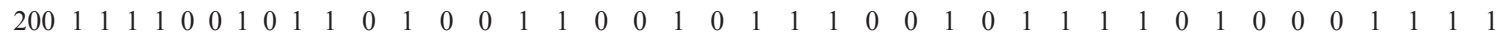

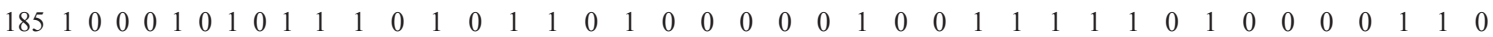
$\begin{array}{lllllllllllllllllllllllllllllllllllllllllllll}160 & 0 & 1 & 1 & 1 & 0 & 1 & 0 & 1 & 1 & 1 & 1 & 0 & 0 & 0 & & 1 & 1 & 1 & 0 & 1 & 0 & 1 & 1 & 0 & 0 & 0 & 0 & 1 & 1 & 1 & 1 & 0 & 0 & 0 & 1 & 0 & 1 & 0 & 1 & 1\end{array}$ $1250 \begin{array}{llllllllllllllllllllllllllllllllllllllllllllll} & 0 & 0 & 0 & 1 & 1 & 0 & 0 & 0 & 0 & 1 & 0 & 0 & 1 & 0 & 0 & 1 & 1 & 0 & 1 & 0 & 0 & 0 & 1 & 0 & 0 & 0 & 0 & 1 & 1 & 1 & 0 & 0 & 1 & 0 & 0 & 1 & 0 & 0 & 0\end{array}$ $100 \begin{array}{llllllllllllllllllllllllllllllllllllllllllll} & 0 & 0 & 0 & 1 & 1 & 0 & 0 & 0 & 0 & 0 & 0 & 0 & 0 & 0 & 0 & 0 & 0 & 0 & 0 & 0 & 0 & 0 & 0 & 0 & 0 & 0 & 0 & 0 & 0 & 0 & 1 & 0 & 0 & 0 & 0 & 0 & 0 & 0 & 1\end{array}$ $\begin{array}{lllllllllllllllllllllllllllllllllllllllllllll}90 & 0 & 0 & 0 & 0 & 0 & 0 & 0 & 0 & 0 & 0 & 0 & 0 & 0 & 0 & 0 & 0 & 0 & 0 & 0 & 0 & 0 & 0 & 0 & 0 & 1 & 0 & 0 & 0 & 0 & 0 & 0 & 0 & 0 & 0 & 0 & 1 & 0 & 0 & 0 & 0\end{array}$ 


\section{Conclusion}

In conclusion, the present study showed reliable amount of genetic variation which exists among rice accessions can be utilized efficiently to select parent for improving cold tolerance. The rice genotypes Giza 177, Sakha 102, Sakha 104, CT6749-36-7-2-M-M, Milyang 80, PSRM1-174B-13 and Ryong Sung 25 showed good performance in their ability to cold tolerance, so they could be used in initiated the breeding program for growing at low temperature levels under Egyptian conditions. Furthermore, due to most of morphological traits are strongly associated with the four SSR markers namely RM 265, RM324, RM163 and RM270, so it could be use these markers as simple tools for selection in the early generation during the applied breeding programme.

\section{$\underline{\text { References }}$}

Abd El-Maksoud, M. M., Abd El-Hadi, A. A., Draz, A. E. and Elgamal, W. H. (2007) Genetical analysis of anther culture response in rice under drought stress. Journal of Agricultural Sci. Mansoura Univ., 32 (4), $2499-2509$.

Andaya V.C. and Mackill D.J. (2003) Mapping of QTLs associated with cold tolerance during the vegetative stage in rice. J. Exp. Bot. 54, 2579-2585.

Anderberg, M. R. (1973) Cluster Analysis for Applications. Academic Press Inc. New York.

Boyer J.S. (1982)Plant productivity and environment. Science, 218, 443-448.

Elgamal, W.H. (2013) Inheritance of some traits related to drought tolerance in rice. PhD. Thesis, Fac. of Agricultural, Mansoura Univ., Egypt.

Elgamal, W. H., Draz A. E., Abd El-Maksoud, M. M. and Elmoghazy, A. M. (2015) Genetic and molecular analysis of rice yield and some anatomical root traits under water deficit conditions. Egypt. J. Agric. Res. 93, 2 (A),315-333.

Glaszmann, J.C., Kaw, R.N. and Khush, G.S. (1990) Genetic divergence among cold-tolerant rices (Oryza sativa L.). Euphytica, 45, 95-104.

Gomez, K. A. and Gomez, A. A. (1984) Statistical Procedures for Agricultural Research, John Wiley $\&$ Sons.

Jiang, W., Jin, Y.M., Lee, J., Lee, K.I., Piao, R., Han, L., Shin, J.C., Jin, R.D., Cao, T., Pan, H.Y., Du, X. and Koh, H.J. (2011) Quantitative Trait Loci for Cold Tolerance of Rice Recombinant Inbred Lines in Low Temperature Environments. Molecules and Cells, Springer, DOI/10.1007/s10059-011-0186-4

Khush G.S. (1999) Green revolution: preparing for the 21 st century. Genome, 42,646-655.

Kim, S., Andaya, V.C. and Tai, T.H. (2011) Cold sensitivity in rice (Oryza sativa L.) is strongly correlated with a naturally occurring I99V mutation in the multifunctional glutathione transferase isoenzyme GSTZ2. Biochem. J. 435, 373-380.

Lee, M. H. (2001) Low temperature tolerance in rice: the Korean experience. In: Increased lowland rice production in the Mekong region, Proceedings of an International Workshop Held in Vientiane, Laos, pp.109-117.

Mackill, D.J. and Lei, X. (1997) Genetic variation for traits related to temperate adaptation of rice cultivars. Crop Sci. 37, 1340-1346.

Murai, M., Hirose, S., Sato, S. and Takebe, M. (1991) Effects of dwarfing genes from Dee-Geo-Woo-Gen and other varieties on cool temperature tolerance at booting stage in rice. Jpn. J. Breed. 41, 241-254.

Nagasawa, N., Kawamoto, T., Matsunaga, K., Sasaki, T., Nagato, Y. and Hinata, K. (1994) Coldtemperature sensitive mutants at the booting stage of rice. Breeding Sci. 44,53-57.

Qia Y. L., Han L. Z. and An, Y. P. (2004) Molecular mapping of QTLs for cold tolerance at the budburst period in rice. Agric Sci China, 3(11), 801-806.

Renata, P.C., Ismael, T. L. and Cabreira, C. (2010) Inheritance of rice cold tolerance at the seedling stage. Sci. Agric. 67(6), 669-674.

Rogers, O.S. and Bendich, A.J. (1988) Extraction of DNA from plant tissues. Plant Mol. Biol. Manual A6 $1-10$.

Saito, K., Miura, K., Nagano, K., Hayano-Saito, Y., Araki, H. and Kato, A. (2001) Identification of two closely linked quantitative trait loci for cold tolerance on chromosome 4 of rice and their association with anther length. Theor. Appl. Genet. 103, 862-868.

Saitou, N., and Nei, M. (1987) The neighborjoining method: a new method for reconstructing phylogenetic trees. Molecular Biology and Evolution, 4, 406-425.

Sanghera, G.S., Hussaini, A. M., Anwer, A. and Kashyap, S.C. (2011) Evaluation of some IRCTN rice genotypes for cold tolerance and leaf blast 
disease undertemperate Kashmir conditions. Journal of Hill Agriculture, 2 (1), 28-32.

Suh, J.P., Cho, Y.C., Lee, J. H., Lee, S.B., Jung, J.Y., Choi, I.S., Kim, M.K., Kim, C.K. and Jena, K.K. (2013) SSR Analysis of Genetic Diversity and Cold Tolerance in Temperate Rice Germplasm. Plant Breed. Biotech. 1 (2),103-110.

Suh, J.P., Choi, Y.H., Kim, K.J., Cho, Y.C., Kwon, S.J., Jeong, Y.P., Jeung, J.U., Choi, I.S., Kim, Y.G., Choi, H.C. and Hwang, H.G. (2004) Genetic diversity and QTLs for grain quality in Japonica rice. Korean J. Breed. 36, 31-37.

Thenmozhi, P. and Rajasekaran, C. (2013) Genetic Diversity and Relationship among 40 Rice Accessions from North-Eastern Zone of Tamil Nadu Using Morphological and SSR Markers. Res. J. Biotech, 8 (9), 32-41.
Xu L.M., Zhou L., Zeng Y.W.; Wang, F.M., Zhang, H.L., Shen, S.Q. and Li, Z.C. (2008) Identification and mapping of quantitative trait loci for cold tolerance at the booting stage in a Japonica rice near-isogenic line. Plant Sci. 174, 340-347.

Yang, Z., Huang, D., Tang, W., Zheng, Y., Liang, K., et al. (2013) Mapping of Quantitative Trait Loci Underlying Cold Tolerance in Rice Seedlings via High Throughput Sequencing of Pooled Extremes. PLOS ONE, 8 (7), e68433. doi:10.1371

Ye, C., Fukai, S., Godwin, I.D., Koh, H., Reinke, R., Zhou, Y., Lambrides, C., Jiang, W., Snell, P. and Redona, E. (2010) A QTL controlling low temperature induced spikelet sterility at booting stage in rice. Euphytica, 176, 291-301.

(Received: $30 / 4 / 2018$; accepted: $18 / 7 / 2018)$

\section{التنوع الوراثى لتحمل البرودة على الأرز فى مرحلة البادرة تحت الظروف المصرية \\ وليد حسن الجمل ، محمود عبد الله على السيد ، عصام عادل ذكى الثامى و جلال بكر أنيس لأليس

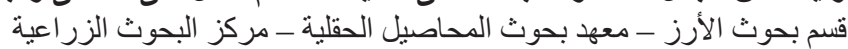

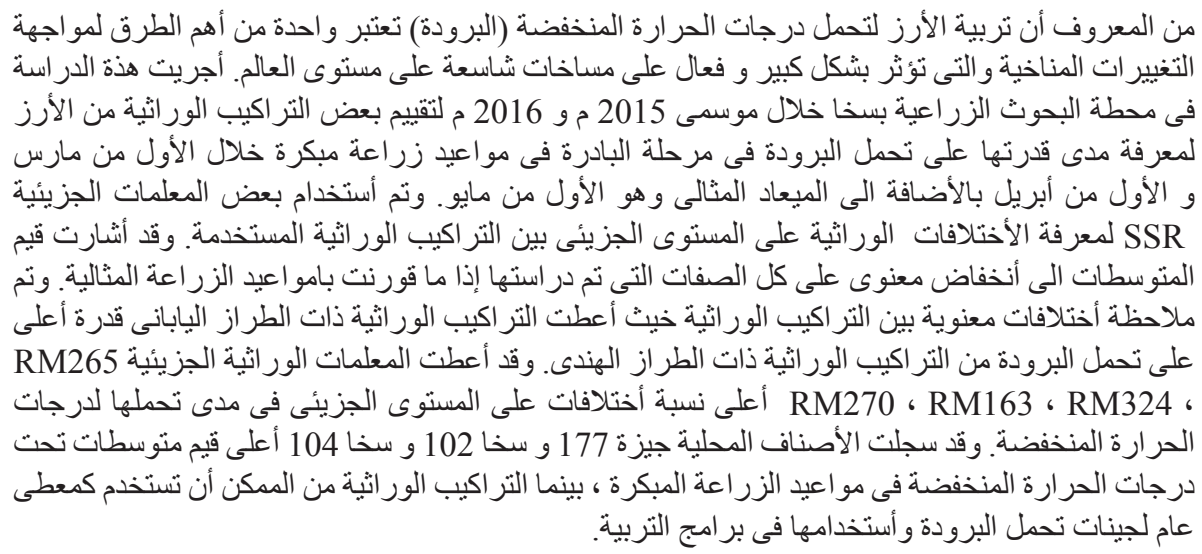

